\title{
Food-Dependent Exercise-Induced Anaphylaxis: A Case Related to Chickpea Ingestion and Review
}

\author{
Chet G. Wong, HonBSc and Sean R. Mace, MB, BCh, FRCPC
}

\begin{abstract}
Food-dependent exercise-induced anaphylaxis (FDEIA) is recognized as a distinct category of exercise-induced anaphylaxis (EIA) but is very likely underdiagnosed. This report describes a 41-year-old Indian woman who experienced two separate episodes of anaphylaxis while dancing after she had eaten chickpea-containing foods. The chickpea, a small legume, is a staple ingredient in culinary traditions from around the world, especially in India, the Middle East, and North Africa. Chickpea-containing dishes are also becoming more widespread in the Western world with the growing popularity of South Asian, Middle Eastern, and African cuisines. It is important to consider FDEIA in cases of unexplained anaphylaxis as reactions can occur several hours after ingesting the culprit food(s). Furthermore, no reaction occurs if a sensitized individual eats the culprit food(s) without exercising afterward; therefore, triggering foods can easily be overlooked. Current ideas on the pathophysiology, predisposing factors, workup, and treatment of FDEIA are also summarized here.
\end{abstract}

Key words: anaphylaxis, exercise, food allergy

$\Gamma$ ood-dependent exercise-induced anaphylaxis (FDEIA) $F$ is a type of exercise-induced anaphylaxis (EIA) that occurs only when a sensitized individual ingests a food allergen(s) and proceeds to exercise within a certain window of time; neither the food(s) alone nor exercise alone is sufficient to induce a reaction. Typical symptoms of EIA include warmth, flushing, generalized pruritis, urticaria, angioedema, wheezing, and, in severe cases, airway obstruction and anaphylactic shock. ${ }^{1}$ FDEIA can be a difficult diagnosis to make because the association between the inciting food allergen(s) and physical activity is not always readily apparent; other conditions that share similar clinical features include food allergy, EIA, asthma, cholinergic urticaria, and laryngospasm. The following case report describes an individual who developed EIA after eating chickpeas and participating in bhangra dancing, a spirited form of modern dance requiring significant energy and stamina with origins in the harvest dances from Punjab. ${ }^{2}$

Chet G. Wong: School of Medicine, Queen's University, Kingston, ON; Sean R. Mace: Division of Allergy and Clinical Immunology, St. Michael's Hospital, University of Toronto, Toronto, ON.

Correspondence to: Dr. Sean R. Mace, 2130 Lawrence Avenue East, Suite 301, Scarborough, ON M1R 3A6.

DOI 10.2310/7480.2007.00011

\section{Case Report}

The patient was a 41-year-old Indian saleswoman who works from home and was referred to the allergy clinic because of a suspected chickpea allergy. She reported two episodes of anaphylaxis associated with the ingestion of chickpeas. The first occurred during a party in the summer of 2005; the patient ate a dish containing chickpea flour at approximately $7 \mathrm{pm}$, and within half an hour, she developed generalized urticaria with facial swelling, mostly in the periorbital region, while dancing. She did not experience any dysphagia, wheezing, or breathing difficulties. At midnight that same evening, the patient felt lightheaded and, after standing up, lost consciousness for less than 30 seconds. After regaining consciousness, she had some nausea and vomiting and felt weak. The patient had not been drinking alcohol at the party and had only Coca-Cola to drink. None of the other guests at the party experienced these symptoms. The patient subsequently went out dancing on other nights without eating chickpeas and was unaffected. Similarly, she also ate chickpeas on several occasions without dancing afterward and did not have any problems. Then, approximately 1 month after her first reaction, she had another reaction that occurred after she had eaten chickpeas and gone dancing.

The patient's past medical history is significant for hypothyroidism. She does not have any known allergies to foods or medications, nor does she have any history of 
eczema or asthma, but she does have seasonal allergic rhinoconjunctivitis. The patient drinks alcohol occasionally and does not smoke. Her family history of atopy includes a sister who has environmental allergies and a 14year-old son who had a childhood allergy to peanuts, lentils, and other legumes; he outgrew most of these allergies but remains allergic to chickpeas. The patient's medications consist of levothyroxine and an oral contraceptive pill.

A complete physical examination was unremarkable, and the patient underwent skin-prick testing for chickpeas and other members of the legume family, including peanuts, peas, green peas, string beans, and soy; she had a positive reaction only to chickpeas. A radioallergosorbent test (RAST) for immunoglobulin E (IgE) antibodies against chickpeas showed a low titre of $0.47 \mathrm{kU} / \mathrm{L}$. This was followed by a supervised graded oral challenge, and the patient tolerated three chickpeas without any consequences.

\section{Discussion}

EIA is a well-known phenomenon, ${ }^{3}$ and FDEIA has been recognized as a distinct variant of EIA for almost 30 years, ${ }^{4}$ although it is less well understood. In fact, the first case report of EIA in 1979 described an individual who had ingested shellfish prior to long-distance running and subsequently developed anaphylaxis. ${ }^{5}$ The true prevalence and incidence of FDEIA are currently unknown as many cases remain undiagnosed. FDEIA has not been found to have a gender or racial bias to date.

There are two types of FDEIA: unspecific FDEIA and specific FDEIA. ${ }^{6}$ Unspecific FDEIA occurs when a susceptible individual exercises after filling his or her stomach, regardless of what has been eaten. In specific FDEIA, the combination of the culprit food antigen(s) and exercise lowers mast cell degranulation thresholds, leading to histamine release and anaphylaxis. ${ }^{7}$ Thus far, the most common food implicated in specific FDEIA is wheat, and molecular studies have identified sequential epitopes in the repetitive domain of wheat gliadins that are bound by IgE antibodies in wheat-dependent EIA. ${ }^{8,9}$ Other triggering foods in FDEIA include buckwheat, pistachios, apples, oranges, corn, mushrooms, and celery. ${ }^{10-16}$

The exact pathophysiology of FDEIA has yet to be determined, but gut permeability is one factor that may play an important role in the development of FDEIA. ${ }^{7}$ An animal study by Yano and colleagues using lysozymesensitized mice revealed that exercise increased the gastrointestinal absorption of lysozyme ingested prior to activity. ${ }^{17}$ Interestingly, sensitized mice were found to have a greater number of mucosal lesions in the small intestine after exercise than sensitized mice at rest or unsensitized mice. Although the mechanism of mucosal damage was not addressed, the authors noted that exercise can cause significant decreases in mesenteric blood flow and that intestinal ischemia has been linked to increased bacterial translocation and absorption of endotoxin from the gastrointestinal tract. These observations suggest that mesenteric ischemia could be responsible for failure of the gastrointestinal mucosal barrier and result in increased absorption of food allergens into the bloodstream.

Another factor hypothesized to be involved with the pathophysiology of FDEIA is tissue transglutaminase (tTG) activity beneath gastrointestinal epithelium. ${ }^{7}$ Palosuo and colleagues showed that tTG cross-links wheat gliadins in individuals with wheat-dependent EIA, causing the formation of high-molecular-weight complexes with enhanced IgE binding capability. ${ }^{18}$ Skeletal muscle contraction during exercise also increases circulating levels of tumour necrosis factor $\alpha$, interleukin- 6 , and glucocorticoid hormones, all of which stimulate tTG activity. ${ }^{18}$ Thus, exercise may activate tTG and increase modification of food-derived peptides, leading to more $\operatorname{IgE}$ cross-linking and mast cell degranulation. However, whether tTG acts on other food substrates besides gliadin remains to be seen.

An important consideration in FDEIA is the relationship between the amount of food allergen ingested and the propensity for an attack. A case report on a young Japanese woman with wheat-dependent EIA by Hanakawa and colleagues described a dose-dependent effect of wheat ingestion on precipitating an allergic reaction. ${ }^{19}$ Another issue of concern is whether medications can influence the onset of FDEIA. Drug-dependent EIA has been reported in a patient on a nonsteroidal anti-inflammatory drug (NSAID), ${ }^{20}$ and several Japanese studies have found that aspirin potentiates FDEIA. ${ }^{8,21,22}$ In addition, Matsuo and colleagues demonstrated that aspirin increased circulating levels of gliadin in patients with wheat-dependent EIA who were fed wheat. ${ }^{8}$ Notably, circulating levels of gliadin correlated with FDEIA symptomatology, supporting the belief that FDEIA is dose dependent. Taken together, these findings indicate that aspirin use may increase gastrointestinal absorption of food allergens and exacerbate FDEIA.

A reasonable workup for FDEIA would include a complete history and physical examination, skin-prick testing, prick + prick testing, applicable RASTs, and foodexercise provocation testing. With regard to prick tests, an 
Italian study on 54 patients with FDEIA recommended that those with suspected FDEIA be skin prick tested using a large panel of foods as patients can have multiple food triggers. ${ }^{23}$ However, this study also revealed that skin-prick testing, prick + prick testing, and RASTs had variable sensitivities for diagnosing FDEIA, depending on what the culprit food was, although in virtually every case, at least one of the three tests was positive for the suspect food. Furthermore, each of the three tests found positivities undetected by the others.

Another study by Harada and colleagues described four patients with wheat-dependent EIA confirmed by prick tests, immunoblot tests, and provocation testing who had negative gluten RAST scores. ${ }^{24}$ In the case reported here, the patient had a positive skin-prick test against chickpeas, which corroborates her history. Even though her chickpea RAST score was low, this does not preclude a diagnosis of chickpea-dependent EIA because there are currently no data regarding the sensitivity of the chickpea RAST for identifying such a case.

A formal double-blind placebo-controlled food challenge (DBPCFC) was not performed in this case. However, the patient's history indicated that she was able to tolerate normal-sized portions of chickpeas without any symptoms as long as she did not engage in vigorous activity afterward. There is no universally accepted protocol for administering a DBPCFC, but such a challenge would involve giving the patient increasing quantities of the suspected food allergen or placebo in the form of an opaque pill or disguised in a liquid medium over 1.5 to 2 hours; if the patient tolerates the equivalent of $10 \mathrm{~g}$ of the dehydrated food without a reaction, it is highly unlikely that the patient has a food allergy. ${ }^{25}$

To determine whether a suspected food is responsible for FDEIA, it is helpful to do a food-exercise provocation test. Again, there is no standardized protocol for provocation testing in cases of suspected FDEIA, but such a test would involve administering increasing amounts of the suspected food at regular intervals and then exercising the subject after each ingestion, with appropriate supervision and resuscitation equipment ready. However, one must keep in mind that some patients with FDEIA do not become symptomatic until several hours later, so a negative provocation test does not rule out the diagnosis. The patient described in this case report declined to return for provocation testing as she was satisfied with our management plan, which included avoidance of chickpeas and chickpea-containing foods prior to vigorous activity and having an epinephrine autoinjector with her at all times.
As mentioned above, the mainstay of treatment for FDEIA is avoidance; the time course from ingestion of food allergen to development of a reaction has not been established definitively, but the Italian study by Romano and colleagues found that sensitized individuals did not have reactions as long as they avoided foods associated with a positive skin test and/or RAST for at least 4 hours prior to exercising. ${ }^{23}$ In the case reported here, the patient lost consciousness briefly and then awoke with nausea and vomiting approximately 5 hours after ingesting the triggering agent. We believe that these symptoms were part of her reaction, and a recent report in the literature describes a similar patient with WDEIA who experienced loss of consciousness and severe anaphylaxis 5 hours after wheat ingestion and exercise. ${ }^{26}$ Of course, total avoidance of suspected foods would be ideal. It would also be prudent for sensitized individuals to avoid NSAIDs and aspirin if they do plan on ingesting suspected food allergens. Patients with FDEIA should be advised to carry an epinephrine autoinjector with them at all times. In an acute attack, antihistamines, corticosteroids, and/or epinephrine can be used depending on the severity of the symptoms. Currently, there are no approved prophylactic agents for FDEIA, although case reports have described various possibilities including sodium bicarbonate, disodium cromoglycate, and terfenadine. ${ }^{27-29}$

The patient described in this report was given a prescription for an epinephrine autoinjector and instructed to avoid eating chickpea-containing dishes prior to dancing or engaging in vigorous physical exercise. She has not experienced another episode since and continues to enjoy her usual foods and activities.

\section{References}

1. Volcheck GW, Li JT. Exercise-induced urticaria and anaphylaxis. Mayo Clin Proc 1997;72:140-7.

2. What is bhangra? Available at: http://www.dholrhythms.com/ index.php?section $=2$ (accessed November 10, 2006).

3. Castells MC, Horan RF, Sheffer AL. Exercise-induced anaphylaxis. Curr Allergy Asthma Rep 2003;3:15-21.

4. Kidd JM, Cohen SH, Sosman AJ, Fink JN. Food-dependent exercise-induced anaphylaxis. J Allergy Clin Immunol 1983;71: 407-11.

5. Maulitz RM, Pratt DS, Schocket AL. Exercise-induced anaphylactic reaction to shellfish. J Allergy Clin Immunol 1979;63:433-4.

6. Chong SU, Worm M, Zuberbier T. Role of adverse reactions to food in urticaria and exercise-induced anaphylaxis. Int Arch Allergy Immunol 2002;129:19-26.

7. Tewari A, Du Toit G, Lack G. The difficulties of diagnosing fooddependent exercise-induced anaphylaxis in childhood-a case study and review. Pediatr Allergy Immunol 2006;17:157-60. 
8. Matsuo H, Morimoto K, Akaki T, et al. Exercise and aspirin increase levels of circulating gliadin peptides in patients with wheat-dependent exercise-induced anaphylaxis. Clin Exp Allergy 2005;35:461-6.

9. Battais F, Mothes T, Moneret-Vautrin DA, et al. Identification of IgE-binding epitopes on gliadins for patients with food allergy to wheat. Allergy 2005;60:815-21.

10. Noma T, Yoshizawa I, Ogawa N, et al. Fatal buckwheat dependent exercise-induced anaphylaxis. Asian Pac J Allergy Immunol 2001; 19:283-6.

11. Porcel S, Sanchez AB, Rodriguez E, et al. Food-dependent exerciseinduced anaphylaxis to pistachio. J Investig Allergol Clin Immunol 2006;16:71-3.

12. Morimoto K, Hara T, Hide M. Food-dependent exercise-induced anaphylaxis due to ingestion of apple. J Dermatol 2005;32:62-3.

13. Morimoto K, Tanaka T, Sugita Y, Hide M. Food-dependent exercise-induced anaphylaxis due to ingestion of orange. Acta Derm Venereol 2004;84:152-3.

14. Pauls JD, Cross D. Food-dependent exercise-induced anaphylaxis to corn. J Allergy Clin Immunol 1998;101:853-4.

15. Okano M, Sakuma Y. Food-dependent exercise-induced anaphylaxis due to matsutake mushrooms. Br J Dermatol 1997;136:805.

16. Silverstein SR, Frommer DA, Dobozin B, Rosen P. Celerydependent exercise-induced anaphylaxis. J Emerg Med 1986;4: 195-9.

17. Yano H, Kato Y, Matsuda T. Acute exercise induces gastrointestinal leakage of allergen in lysozyme-sensitized mice. Eur J Appl Physiol 2002;87:358-64.

18. Palosuo K, Varjonen E, Nurkkala J, et al. Transglutaminasemediated cross-linking of a peptic fraction of $\omega-5$ gliadin enhances IgE reactivity in wheat-dependent, exercise-induced anaphylaxis. J Allergy Clin Immunol 2003;111:1386-92.
19. Hanakawa Y, Tohyama M, Shirakata Y, et al. Food-dependent exercise-induced anaphylaxis: a case related to the amount of food allergen ingested. Br J Dermatol 1998;138:898-900.

20. van Wijk RG, de Groot H, Bogaard JM. Drug-dependent exerciseinduced anaphylaxis. Allergy 1995;50:992-4.

21. Harada S, Horikawa T, Ashida M, et al. Aspirin enhances the induction of type I allergic symptoms when combined with food and exercise in patients with food-dependent exercise-induced anaphylaxis. Br J Dermatol 2001;145:336-9.

22. Aihara M, Miyazawa M, Osuna $\mathrm{H}$, et al. Food-dependent exerciseinduced anaphylaxis: influence of concurrent aspirin administration on skin testing and provocation. Br J Dermatol 2002;146:46672.

23. Romano A, Di Fonso M, Giuffreda F, et al. Food-dependent exercise-induced anaphylaxis: clinical and laboratory findings in 54 subjects. Int Arch Allergy Immunol 2001;125:264-72.

24. Harada S, Iijima M, Nakamura A, et al. Four cases of wheatdependent exercise-induced anaphylaxis with negative gluten CAPRAST scores. Jpn J Allergol 2007;56:41-8.

25. Sampson HA. Use of food-challenge tests in children. Lancet 2001; 358:1832-3.

26. Oyefara BI, Bahna SL. Delayed food-dependent, exercise-induced anaphylaxis. Allergy Asthma Proc 2007;28:64-6.

27. Katsunuma T, Iikura $\mathrm{Y}$, Akasawa A, et al. Wheat-dependent exercise-induced anaphylaxis: inhibition by sodium bicarbonate. Ann Allergy 1992;68:184-8.

28. Juji F, Suko M. Effectiveness of disodium cromoglycate in fooddependent, exercise-induced anaphylaxis: a case report. Ann Allergy 1994;72:452-4.

29. Fujimoto S, Kurihara N, Hirata K, et al. Successful prophylaxis of wheat-dependent exercise-induced anaphylaxis with terfenadine. Intern Med 1995;34:654-6. 